\title{
Analytical lights and shadows in metabolomics research on obstructive sleep apnea
}

\author{
Castillo-Peinado LS $S^{1,2,3,4}$, Jurado-Gámez B ${ }^{1}$, Luque de Castro MD 1,2,3,4* \\ ${ }^{1}$ Maimónides Institute of Biomedical Research (IMIBIC), Reina Sofía University Hospital, University of Córdoba, Córdoba, Spain \\ ${ }^{2}$ Department of Analytical Chemistry, Annex Marie Curie Building, Campus of Rabanales, University of Córdoba, Córdoba, Spain \\ ${ }^{3}$ University of Córdoba Agroalimentary Excellence Campus, ceiA3, Córdoba, Spain \\ ${ }^{4}$ CIBER Fragilidad y Envejecimiento Saludable (CIBERfes), Instituto de Salud Carlos III, Spain
}

\begin{abstract}
A critical opinion about how clinical researchers face to the metabolomics-obstructive sleep apnea (OSA) binomial is here presented from the point of view of analytical chemists. Thus, positive and negative (lights and shadows) aspects related to the types of samples used for the target studies, the pros and cons of the devices used for sampling and present storage conditions, and actions for their improvement are expressed. Sample preparation and its multiple facets that required to be clarified are discussed, as well as those related to analysis. A key aspect as data treatment has been discussed in the light of the cohorts handled to obtain the information to be subjected to appropriate/inappropriate treatment, and support on suitable examples has been provided. The search for OSA biomarkers, one of the areas to which key investigations are devoted at present, is critically discussed by showing the weak points and proposing new ways for improvement. A final section is devoted to aspects that may help to improve metabolomics-OSA research, a field asking for more attention.
\end{abstract}

\begin{abstract}
Abbreviations: AECOPD: Acute exacerbations of chronic obstructive pulmonary disease, AHI: Apnea-hypopnea-index (apnoea-hypopnoea-index), AUC: Area under the curve, BCAAs: Branched-chain amino acids, CPAP: Continuous positive airway pressure, COPD: Chronic obstructive pulmonary disease, EB: Exhaled breath, EBC: Exhaled breath condensate, ECL: External cavity laser, EDS: Excessive daytime sleepiness, FENO: Fraction of nitric oxide in expired gas, FI: Flow injection, GC: Gas chromatography, GC-MS: Chromatography coupled to quadrupole mass-spectrometry, GC-TOF/ MS: Gas chromatography coupled to time-of-flight mass spectrometry, HOMA-IR index: Homeostatic model assessment for insulin resistance, LC-QTOF/MS: Liquid chromatography-quadrupole time of flight-mass spectrometry, LC-TOF/MS: Liquid chromatographymass spectrometry, LC-MS/MS: Liquid chromatography coupled with tandem mass spectrometry, mRNA: Messenger RNA, NMR: Nuclear magnetic resonance, QCs: Quality controls, OA CEAS: Off axis cavity enhanced absorption spectroscopy, OSA: Obstructive sleep apnea (obstructive sleep apnoea), PLS-DA: partial least squares discriminant analysis, PSG: Polysomnography, ROC: Receiver-operator characteristic, SESI-MS: Secondary-electrospray-ionization-mass spectrometry, SIL-ISs: Stable isotopic labeled internal standards, TD-GC-MS: Thermal desorption gas chromatography mass spectrometry, TRP: Tryptophan, VOCs: Volatile organic compounds
\end{abstract}

\section{Introduction}

Obstructive sleep apnea (OSA) is characterized by episodes of partial or complete pharyngeal collapse associated with reduction (hypopnea) or total (apnea) absence of airflow during sleep that result in intermittent hypoxia, hypercapnia and sleep disruption.

Obesity could increase the likelihood of airway collapse by directly affecting the anatomy of upper airway as fat is deposited on surrounding structures. OSA symptoms include habitual snoring, witnessed apneas, and reporting of disturbed unrefreshing sleep, frequently accompanied by excessive daytime sleepiness, and daytime neurobehavioral problems.

OSA is defined as the occurrence of more than five obstructive events per hour-apnea-hypopnea-index (AHI) $>5 /$ h-associated with typical symptoms. The estimated prevalence of moderate to severe OSA (AHI $\geq 15 / \mathrm{h}$ ) is $10-17 \%$ in adult men and $3-9 \%$ in adult women with a higher prevalence in adults $>50$ years of age [1]. The percentage within each gender interval seems to be influenced by the ethnia [2].

OSA has adverse metabolic consequences in the pediatric population and several pathways have been suggested for the interaction between obesity, metabolic dysfunction and OSA $[3,4]$. The risk of OSA is higher in obese children, and OSA may in turn worsen obesity [5-7].

The gold-standard diagnostic test for OSA is labor- and timeintensive in laboratory polysomnography (PSG) [8,9]. Nocturnal continuous positive airway pressure (CPAP) therapy seems to be the most effective treatment for OSA. A CPAP device consists of a unit that generates airflow, which is directed to the airway via a mask and prevents upper airway collapse; thus, it is presumed to reverse the pathophysiological consequences of OSA. Nevertheless, the association of OSA to many chronic diseases such as hypertension [10], myocardial

${ }^{*}$ Correspondence to: M.D. Luque de Castro, Department of Analytical Chemistry, Annex Marie Curie Building, Campus of Rabanales, University of Córdoba, Córdoba, Spain; E-mail: qallucam@uco.es

Key words: Metabolomics, Obstructive sleep apnea, Sample preparation, Data treatment, Biomarkers

Received: November 08, 2020; Accepted: November 12, 2020; Published: November 26, 2020 
infarction [11,12], stroke [13,14], insulin resistance [15-18], diabetes [19], nonalcoholic fatty liver disease [20], postmenopausal status in women and craniofacial structure [21] and, most recently, mild cognitive impairment (MCI)/dementia [22] and cancer [23] makes adherence to CPAP each time more limited despite the efforts to improve comfort [24-26]. For this reason, OSA lends itself to a personalized approach to diagnosis and therapy after checking that different clinical OSA subtypes likely benefit from different therapies. Hypoglossal nerve stimulation, radiofrequency ablation of the soft palate, oral appliances or surgical procedures are useful second-line therapy in patients who cannot tolerate CPAP devices. Of great interest are the technological advances that allow patients to participate in their own care, thus improving CPAP compliance. Interestingly, the foreseeable future involves focusing the efforts on prediction and prevention of OSA at an individual level, in which P4 medicine is being to play a key role [27]. The four Ps offer a means to: Predict who will develop disease and co-morbidities; Prevent rather than react to disease; Personalize diagnosis and treatment; have patients Participate in their own care. The fact that each OSA patient has a different pathway to disease, and its consequences makes P4 medicine a conceptual framework that provides the basis for a new approach to OSA that may predict and prevent those at high risk for OSA and consequences, personalize the diagnosis and treatment of OSA and build in patient participation to manage OSA.

The differences in OSA subtypes have been related to genetic, epigenetic and environmental differences among individuals [27]. On the other hand, genetic studies suggest that OSA cannot represent a simple condition associated to a few genes or proteins, but a manifestation of multiple interconnected pathways and numerous molecular abnormalities [28] that justifies its close relationship with a number of diseases. This behavior leads to consider that the genetic and epigenetic changes (even environmental changes) must be downstream reflected in proteins and finally in metabolites changes. The gut microbiota seems also to be related with OSA, providing key information on both the disease itself and its evolution during treatment [29].

The role of metabolomics-the younger of the great omics-in the research on OSA has been scant and it has not been always well understood by the authors working in this field, who have considered this discipline as "a method to explore novel biomarkers and identify physiological and pathological mechanistic processes" [30], as "the field of micromolecules" [31], or even they have ignored metabolomics as such discipline considering it only as a method to obtain metabolic profiles [28]. Most of this research has been focused on the search for metabolites that can act as new biomarkers for OSA with sensitivity and selectivity as high as possible; that is, compounds that experience high changes in concentration in individuals affected by OSA, but no in those affected by other diseases.

This article constitutes an attempt of discussing the present state of metabolomics in OSA research from an analytical point of view, and clarifying analytical concepts not always well understood by medical researchers.

\section{Types of samples, collection devices and storage conditions}

As commented before, most of the studies on the binomial metabolomics-OSA have been focused on the search for biomarkers of this disease, and most of the common clinical samples have been used with this purpose.

Blood (as either serum or plasma) is the most common sample used in metabolomics studies in general and in the look for biomarkers in particular, despite some authors complain about the high concentration of proteins in this biofluid [32]. The authors do not justify, most times, the use of serum or plasma for their studies, and they rarely make a comparison of the results provided by both types of blood samples [33]. This comparison would assure the suitability of the sample for OSA studies. In addition, once the type of blood-derived sample has been selected, the influence of the collection tube should be studied taken into account that a number of metabolomics changes are clearly influenced by this device [34] and also the stability of the sample (e.g., storage temperature, number of freeze/thaw cycles) should be a matter of research [35].

Urine is considered for some authors as almost the sample panacea for clinical studies in general and for metabolomics studies in particular. As main advantages they include, (i) ease of sample collection-there is no requirement of trained staff for collection; (ii) noninvasiveness of the collection procedure; (iii) availability in large amounts; (iv) relative chemical and physical stability; and (v) less complex nature as compared to blood [36]. Disadvantages no considered by enthusiastic of urine as clinical sample are the necessity for volume normalization for which a definitive protocol has not been established yet, and the discussed stability of this sample [37]; the added complexity owing to the existence of phase on of proteins by normal healthy subjects-within the range of $0-0.8 \mathrm{~g} / \mathrm{L} \mathrm{[39],} \mathrm{although} \mathrm{this} \mathrm{content} \mathrm{is} \mathrm{estimated} \mathrm{to} \mathrm{be}$ 1000 times lower than in blood [40].

Faecal samples have been scantly used in this field; nevertheless, they can provide a key information on the role of microbiota in OSA, and even on earlier biomarkers of the disease [41]. Studies on the stability of this sample are a pending goal.

Saliva samples (up to $10 \mathrm{~mL}$ ) have been collected by passive drooling of clear saliva and used to look for fatigue biomarkers, mainly peptides, which can be closely related with those for OSA. Taking into account the high enzyme content of this sample, an in depth study on its stability is mandatory to provide appropriate interpretation of the results [42].

Breath is the key sample for the study of lung diseases. For this reason, breath analysis is not a recent matter of research because as far as 1971 the breath profile of 250 volatile organic compounds (VOCs) was obtained after trapping the sample in a cooled stainless-steel tube [43].

Exhaled breath constitutes an accessible sample, obtained in a non-invasive manner, easily repeatable, released spontaneously, and in relatively large amounts. This biofluid is characterized by the presence of exogenous and endogenous compounds, as well as microbiomeassociated metabolites. In addition to VOCs, this sample contains detectable levels of semi-volatile organic compounds (SVOCs), watersoluble metabolites, lipids, other macromolecules such as proteins and DNA, and microbiota components such as bacteria and viruses.

The obvious importance of this sample on the search for lung cancer biomarkers [44-46], and in general for lung diseases, is also applicable to OSA biomarkers. Different types of breath samples can be collected depending on the sampling device (exhaled breath and exhaled breath condensate-EB and EBC, respectively), and on its off- or on-connection with equipment for subsequent steps of the analytical process. All the sampling procedures are relatively rapid, safe and comfortable from a patient perspective; however, their standardization is not yet well established [47], despite methodological recommendations for analysis of EBC and FENO (fraction of nitric oxide in expired gas) were published in 2005 [48,49], also recently 
published for VOCs and particles [50]. The published report clearly established the no intention for providing clinical guidance on disease diagnosis and management. Despite these contributions, studies on optimization and standardization of sampling, storage and pretreatments are still required in this field.

Off-line sampling of EB uses special disposable bags or traps where the sample is stored until analysis. In fact, after sample collection, storage and preconcentration steps precede analysis. Sampling and storage of $\mathrm{EB}$, however, is associated with several problems including degradation of breath compounds and introduction of artifacts or impurities [5153]; therefore, selection of the sampler and storage conditions is crucial in the quality of the subsequent analysis [47]; conditions very different depending on the required analysis: targeted or untargeted.

Only EB allows real-time analysis of breath components, for which a number of samplers have been designed. The samplers allow on-line connection to electronic noses [54-56] or to mass detectors (either, directly or through a chromatograph, mainly a GC), each with its characteristic advantages and disadvantages [57-59].

EBC sampling is always off-line developed as it involves the use of refrigerated bags (usually polyethylene bags at $-20^{\circ} \mathrm{C}$ ) that allow collection of both VOCs and compounds dissolved in the aqueous vapor phase. Commercial portable EBC samplers are the TurboDECCS (that contains a disposable collection cell inserted in a Peltier refrigeration unit and electronic adjustment of the condensation temperature), and the RTube EBC sampler (that consists of a large T-section furnished with a disposable valve and a saliva separator, a collection tube made of polypropylene, and a cooling sleeve that can be refrigerated prior to analysis). The ECOScreen 2 sampler is a no-portable sampler commonly used at present in hospitals and clinical research centers, which allows controlled collection of EBC into two separated bags for physical separation between the air exhaled from the upper-central and distal airways with minimal contamination from saliva [60]. The opinion that "Analyzing non-volatile compounds in the liquid phase of the exhaled breath has the obvious limitation that many VOCs are not captured [47]" is no applicable when the ECOScreen2 sampler is used, as most VOCs are at the liquid state at $-20{ }^{\circ} \mathrm{C}$, the common temperature for sampling $\mathrm{EBC}$. In addition to the wide metabolome profile $\mathrm{EBC}$ provides, other key components of EBC such as proteins and DNA allow to expand the use of this sample to other omics, and thus to integrate omics. The potential of this integration constitutes a key challenge for omics researchers. Nevertheless, it must be emphasized that optimization and standardization of sampling, storage and pretreatment steps are still required to achieve the clinical application of off-line breath analysis.

Breath biopsy is the name given to either $\mathrm{BC}$ or EBC, similarly to the name of liquid biopsy for blood analysis [61], and clearly try to establishing the wide profile of the breath metabolome provided by using the appropriate sampler to obtain the target sample.

The in depth study on EBC developed by the authors, which encompasses sample preparation for subsequent GC-TOF/MS [62] or LC-TOF/MS [63], and application of the developed methods to studies of lung cancer $[45,46,64]$, could be a strong starting point-at present in development -for an in depth application of EBC in OSA studies.

Sweat has so far deserved scant interest in the clinical field in relation to lung diseases. After a review published by the authors' group as a call for attention on this sample [65], some guidelines on what is done and what should be done in the use of sweat as clinical sample [66], and an in depth study on sample preparation prior to LC-MS/
MS [67], then applied to changes in metabolites after moderate exercise [68] and to dry sweat [69], and finally to lung cancer screening, the authors group is ready for the use of sweat to study other lung diseases as OSA. The research the group is developing will also be an invitation to other groups to go inside this subject.

It is remarkable that never a stability study of the target biofluid under the storage conditions has been developed in the research on OSA-metabolomics, despite two stability studies should be mandatory: one of them preceding the research to detect both degradation and potential reactions of the components of the original sample with the degradation products; and the other prior to the proposal of the found biomarkers and devoted specifically to their stability.

\section{Sample preparation}

The crucial influence of sample preparation on the analytical results is well known by analysts [70]. The aim of this key step of the analytical process is to obtain an analytical sample [71] as appropriate as possible for identification, quantitation or semiquantitation of the target analytes. There are many variables involved in sample preparation, the influence of which on this step is a function of the type of sample, the target analyte(s) and the analytical platform in which the prepared sample will be inserted (either the detector or the high resolution equipment for individual separation, if required, prior to detection).

Despite a number of authors working in the OSA-metabolomics field apply correctly the terms involved in this step, also their incorrect use introduces some shadows in the literature on this subject. It is a common mistake to give the name of "sample" to each solution resulting from the sometimes-multiple sub-steps involved in sample preparation. As examples, (i) the solution that results after solid-liquid extraction or leaching is not the original sample, as a part remains as solid (therefore, it is the extract); (ii) the liquid phase resulting from elution after liquid-solid-phase extraction is the eluate; (iii) the fraction of the original sample ready for being analyzed is the analytical sample [71].

The variety of terms involved in this step and their correct use deserve clarification in the light of the most common errors found in the literature on the OSA-metabolomics binomial, which, of course, is applicable to other fields. In the sentence "thawed urine $(1 \mathrm{~mL})$ was mixed with $0.5 \mathrm{~mL}$ of $0.2 \mathrm{M}$ sodium phosphate buffer to control $\mathrm{pH}$ " [71] the correct verb is to adjust as the buffer does not control. On the other hand, the following steps cannot be repeated by a reader interested in reproducing the experiment: "The mixture was placed on ice for 10 minutes and then centrifuged at $7000 \mathrm{~g}$ for 10 minutes. $500 \mu \mathrm{L}$ of the supernatant was extracted and combined with ..." [72]. What was the extraction technique and how the authors applied it?

A common omission also found in the literature on OSAmetabolomics, as in other fields, is about the time the different substeps of sample preparation require, which hinders the readers to decide if either the proposed procedure is adequate for their purposes or it is too much time-consuming. For example, in the procedure "The combined extracts were evaporated to dryness under a gentle stream of nitrogen at $40{ }^{\circ} \mathrm{C}$. After addition of toluene $(20 \mu \mathrm{L})$ and $\mathrm{N}-$ methyl-N(tert-butyldimethylsilyl) trifluoroacetamide $(20 \mu \mathrm{L})$ to the residue, the mixture was heated at $60^{\circ} \mathrm{C}$ for $30 \mathrm{~min}$, allowed to cool and analyzed by GC-MS." [73], the time required for evaporation to dryness should be approximately established, as well as the allowed time to cool. Besides, addition of toluene and a reagent to a solid residue does not become a mixture until dissolution of the residue. 
In dealing with blood, most of the authors never explain how they obtain the plasma or serum sample from the whole blood. As example "Blood samples were collected and the collection tubes were centrifuged and plasma/serum samples were aliquoted and stored at $-70{ }^{\circ} \mathrm{C}$ " [74]. The type of collection tubes used can be a key datum for readers interested in reproducing the results as starting point for a subsequent research. Removal of proteins by precipitation is a mandatory step for subsequent MS or NMR analysis in working with serum; therefore, the conditions for centrifugation (expressed as $g$ value) must be established clearly, as incomplete separation of the precipitate can be the cause of interferences that seriously could modify the results.

The problems involved in providing correct information to the readers are even more relevant when commercial kits are used for sample preparation. The incomplete knowledge about how the target kit works leads to big mistakes in the procedures. This is the case for quantitation of 86 metabolites in urine with no distinction between eluent (the liquid used to elute the analytes retained in a sorbent) and eluate (the eluent containing the eluted analytes). Determination of the analytes in the eluent is an impossible task as it does not contain them [75]. On the other hand, small changes the authors introduce in the procedure with respect to the manufacturer's instructions should be always detailed.

\section{Analysis in OSA-metabolomics}

There are three general aspects that require to be taken into account prior to entering in the analyses involved in OSA-metabolomics: (1) in dealing with calibration, most authors give the name of "calibration curve" to the portion for which they have checked linearity within a given concentration range and calculate a regression coefficient close to 1. (2) Quality controls (QCs) are used hardly to measure the performance and stability of the system under study; only some authors use these controls at the beginning of a run and along the sequence of samples [76]. (3) Despite the use of stable isotopic labeled internal standards (SIL-ISs) is very common in metabolomics [37], this is not the case in OSA-metabolomics, where few studies have involved this tool of paramount importance to assess the correct assignation of signals in MS analysis.

Clinical studies in general and those devoted to OSA-metabolomics in particular are dominated by the use of dedicated analyzers, and the information they provide is sent to the medical researchers by the personnel who attend this type of analytical equipment. An example of this situation is the study of the potential changes in branched-chain amino acid metabolism induced by sleep fragmentation that may suggest a new mechanism linking OSA and glucose homeostasis [77]. With the aim of elucidating if these changes exist, serum glucose was measured using the Architect c16000 platform (Abbott Diagnostics, Abbott Park, IL); serum insulin levels were measured using the Cobas e411 platform (Roche Diagnostics, Germany); HbA1C was quantified by HPLC (no detector was specified, but only the separation equipment) in $\mathrm{K}_{2}$ EDTA-whole blood using the Adams A1C HA-8180 system (Arkray, Japanlucose), and insulin levels were used to estimate insulin resistance using the homeostasis model assessment (HOMA-IR index). Amino acid levels were analyzed using a Biochrom 30+ chromatographic analyzer (Biochrom, UK), of which the authors specify connection to a spectrophotometric detector.

The use of commercial kits is a common place in clinical analysis that involves two main shortcomings: cross-reactions (of which clear examples are the kits for vitamin D metabolites [78]), and high analysis costs. The latter seems to be a no crucial problem as, for example, in the analysis of breath condensate, enzyme immunoassays are presently the most used [47]. When commercial kits are used for quantitation of a high number of metabolites, the quantitative or semiquantitative nature of the results is not clear, and the procedures are difficult to understand even for analytical chemists. Such is the case with the quantitation of 86 metabolites in urine, for which the sample preparation step is discussed in the previous section [75].

The difficulty for reproducing the results found by the authors or for using something similar by other groups is sometimes an impossible task. Such is the case with the publication from Cho et al., [75] in which the results obtained using the proposed kits were validated using a Flow Injection (FI) system online connected to an MS spectrometer. The authors injected an eluent in the FI manifold and used isocratic elution. There is no reference to the use of any separation column that justifies the use of an eluent and the elution in any possible regime. A number of other examples found in the literature show that the high analytical costs of the immunoassays kits are not compensated by good intra-assay and inter-assay variability that is of the order of 8 and $21 \%$, respectively [74].

Electronic noses (e-noses) are specific devices for real-time monitoring of volatiles in very different fields, including military, environmental, food industry, and, obviously, the clinical field (VOCs analysis). Differently from gas GC-MS, e-noses distinguish VOCs spectra by pattern recognition, as they consist of a pre-specified panel of electronic sensors (VOCs of interest, no untargeted). They recognize the combinations of VOCs (specific breath pattern) and thus theoretically a specific disease profile, but do not allow the structural identification of single breath compounds and thus do not provide novel insights into disease mechanisms [79]. The major advantage of enoses is their portability, and their main disadvantage their incapacity for metabolomics studies. Thus, while changes of the e-nose breath pattern after one night of CPAP therapy or those related to comorbid diseases have been shown [80] (and even distinction between OSA patients and those with overlap syndrome COPD/OSA or COPD), breath profiles of patients with an overlapped syndrome could not be clearly separated from those of COPD patients [81]. In short, despite e-noses allow discrimination based on pattern differences, metabolites and mechanisms involved in them constitute information prohibited for being provided by these devices. In short, an e-nose is only able to recognize a simplified EB pattern and cannot detect specific VOCs: never a given metabolite is detected.

MS and nuclear magnetic resonance (NMR) approaches-contrarily to e-noses-enable not only to measure concentrations, but also to identify exhaled compounds. In addition to the gold standard methods for a wide number of metabolites in general, and for those involved in lung diseases in particular, developed using MS detectors in its multiple variants, NMR has provided, and is providing, key contributions in the OSA-metabolomics field. Such is the case with metabolomics discrimination of acute respiratory failure due to COPD exacerbation from respiratory failure due to heart failure and pneumonia using urine and serum as target biofluids [72]. No unique biomarkers were proposed by any of the two types of samples for diagnosis of COPD.

One of the MS approaches for real-time analysis of EB that should be highlighted is SESI-MS, recently applied to patients with OSA [82]. The components of EB are ionized using an electrospray of pure solvent with high ionization efficiencies that allow detection of a broad metabolites profile that encompasses both volatile and nonvolatile components with high molecular weights, which are not covered 
by real-time or off-line breath detectors based on other analytical techniques [82-84]. In addition, it seems that the high resolution of SESI-MS allows precise discrimination of compounds with the same nominal mass but different elemental composition. More research is this field is mandatory to validate the performance of SESI-MS.

Traditional coupling of high-resolution separation equipment with different types of MS detectors has provided information on increased or decreased levels of target metabolites in OSA patients with respect to healthy individuals. Caution with these results must be taken as despite OSA patients-healthy individuals discrimination works correctly; discrimination fails when patients with other diseases instead of healthy individuals are involved in the cohort under study. Thus, studies such as those reporting: (i) increased levels of exhaled pentane-a marker for lipid peroxidation-in OSA patients after sleep, but not in healthy subjects, were found using GC-MS [85]; (ii) exhaled butanol was found to be enhanced in OSA patients compared with healthy subjects using thermal desorption GC-MS [86]; (iii) several VOCs (e.g., isoprene) were found to be increased in the EB of OSA patients using GC-MS [86] (some of these VOCs were shown to correlate with the severity of OSA). The importance of these studies is diluted after knowing that CPAP treatment decreased the levels of exhaled isoprene and acetone; butanol is involved in various biological processes including antiinflammatory regulation, and so on [87].

Comparison of OSA patients before and after CPAP therapy resulted in a distinct breath profile allowing differentiation between treated and untreated OSA with a high diagnostic accuracy in a blind prediction (sensitivity of $92.9 \%$ and specificity of $84.6 \%$ ) based on the change in 62 exhaled features, among which 16 metabolites were identified [88]. Most of the identified molecules stemmed from specific chemical families and they were known human metabolites (e.g., aldehydes, furans). Some of these molecules had previously been reported to be found under conditions of increased physical stress such as exercise or in patients with myocardial infarction. Other molecules have been found in association with oxidative stress and lipid-peroxidation as well as with cytotoxicity. Isoprene, one of the identified discriminating molecules, plays a role in sleep regulation (elevated during sleep, lowest level in the morning after wake-up). In addition to human metabolites, secondary metabolites from the gut microbiome were found increased in untreated OSA, suggesting an altered metabolic interplay with the gut flora in OSA patients. All these data support the association of known pathophysiological consequences in OSA and complicate the diagnostic of this disease.

Collaborations among different medical areas make possible the use of uncommon equipment. Such is the case with the use of a detector based on external cavity laser (ECL)-based off-axis cavity enhanced absorption spectroscopy (OA-CEAS), utilized to measure the concentrations of exhaled carbon dioxide in $\mathrm{EB}$, whereas the levels of exhaled acetone and butanol were determined by thermal desorption GC mass spectrometry (TD-GC-MS) [86]. The very low, ppbv range at which $\mathrm{CO}_{2}$ could be detected did not contribute to the results the study provided. The present frenetic search for diagnosis of COVIC-19 that has promoted the analysis of breath by GC-ion mobility spectrometry [89] is also an example of both the use of uncommon equipment and the omnipresence of breath as sample in lung-disease studies.

\section{Data treatment and results}

It is well documented that clinical researchers are, in general, endowed with a large experience in data treatment; nevertheless, they fail sometimes in application to data treatment in dealing with OSA- metabolomics. Some examples of the lights and shadows concerning treatment of the data provided in this area by the given analytical equipment are as follows.

The first step to obtain representative data for their subsequent treatment is an appropriate selection and classification of the individuals who participate in a given study. Very restrictive criteria for cohort selection should be avoided; except when the interest is focused on a given subgroup, individuals from all the disease stages should be included. An example of a restrictive criterion is that used by Barcelo et al., [77], who studied the effect of OSA on the metabolic system of children aged between 3 and 14 years, particularly focused on branched-chain amino acids (BCAAs) in serum associated to insulin resistance, looking for a mechanism to link OSA and glucose homeostasis [77]. They analyzed continuous variables by the Student's $t$-test, and categorical variables by the Chi-square test, while bivariate associations between BCAA levels polysomnographic and metabolic variables were analyzed by correlation Peasons's coefficients, and multivariate regression analyses were used to assess the relationships between polysomnographic or anthropometric parameters and levels of BCAA. Statistical significance was considered for $p$ values lower than 0.05 . The information the authors achieved suggests the searched mechanism linking OSA and glucose homeostasis through leucine, isoleucine and valine [77]. Despite that metabolism, catabolism, the nature of the compounds (metabolites) found as link in the target mechanism, and the type of developed research, metabolomics as such does not appear in the article. Similarly happens in the article from Scarlata et al. [54] but justified in this case, as they support their study on the analytical information provided by an e-nose.

The results provided by healthy individuals hardly can be extrapolated to patients. This is the case with the study looking for salivary biomarkers of the effect of sleep deprivation with the final aim of facilitating the diagnostic or treatment of sleep disorders [42]. Thirty young healthy adults were selected, 14 of them constitute the control fraction, and the other 16 were sleep deprived. In fact, the authors preselected two metabolites (two peptides) to study the relationship between changes in their ratio with mood changes as a function of sleep deprivation. The difficulty to relate the obtained results with chronic sleep deprivation is clear, to which also contribute inadequate sampling, scant information on the statistical analysis of the data, particularly on the receiver-operator characteristic (ROC) curves.

The involvement of an OSA-patients cohort and comparison with healthy individuals is not a good alternative either, as was the case in the study by Wang et al. [90]. Better was the study by Fortis et al. [72] concerning discrimination of acute respiratory failure due to acute exacerbation of chronic obstructive pulmonary disease (AECOPD) from respiratory failure owing to heart failure and pneumonia as samples from the four types of patients were obtained. Nevertheless, the number of each group was small (between 8 and 15) [72]. The data obtained by NMR from serum and urine were compared by $t$-test, Mann-Whitney, ANOVA, or Kruskal-Wallis with post-hoc Bonferroni and Dunn's test when appropriate for continuous variables and Fischer's exact test for categorical variables. The differences in metabolic profiles were attributed to whether the patient was in respiratory failure or not, but further studies with larger number of samples are mandatory for conclusive results that lead to potential metabolic biomarkers for early diagnosis of AECOPD.

A more complete study also involved NMR to provide data from serum, EBC and urine (85, 82 and 91 samples, respectively) taken with 
a good criterion as over half of the individuals had been diagnosed with cardiovascular disease, including ischemic heart disease and/ or arterial hypertension and/or had suffered a brain stroke [91]. Data treatment involved PLS-DA methodology combined with VIP-scores and SRs for different sets of metabolites aimed at knowing if: (i) a set of metabolites would diagnose a patient with either COPD disease or OSA syndrome, and determine the probability of false positive diagnostic; (ii) the metabolites in a given biofluid suffice for the diagnosis; (iii) the necessity or not of a combination of the metabolites present in two or the three biofluids for correct diagnose of a patient. After AUC estimation of the developed models, the authors concluded that 10 urine metabolites, among the others, presented the highest probability for correctly identifying patients with COPD and the lowest probability for an incorrect identification of the OSA syndrome as developed COPD. The urine metabolites selected by the SR approach provided a model with a specificity of $100 \%$ and a sensitivity of $86.67 \%$, and with a good prediction performance (AUC test $=0.95$ ) in comparison to all of the other models that were constructed. Thus, the no necessity for a combination of two biofluid metabolites or metabolites of all three types of biofluids to obtain a diagnostic model with improved predictive abilities was supported [91]. It must be noted that no control individuals were involved in the cohort.

It should be emphasized that most of the studies on OSAmetabolomics are pilot studies; therefore, as they lack enough number of samples, their results can be only promising. Assessment by a numerous cohort with different levels of OSA penetration, wide age distribution and gender parity would be of great interest. The errors in previous studies should be an always present aspect in new research.

As far as the authors know, there are not studies on OSAs-integrated omics as is the case with asthma, in which levels of metabolites (medium- and long-chain fatty acids) were related with the expression of the enzyme that catalyzes their metabolism [92] by involving a cohort composed by 161 individuals divided in four groups: obese asthma $(n=40)$, obese non-asthma $(n=40)$, non-obese asthma $(n=41)$ and non-obese non-asthma healthy controls $(n=40)$. An integrate treatment of the data from the two omics is missing.

\section{Search for OSA biomarkers}

The definition of a biomarker has gone through multiple evolutionary perspectives. Nowadays, a biomarker would be viewed as a "biological molecule found in blood, other body fluids or tissues that is a sign of normal or abnormal processes or of a condition or disease" [93]. In the context of OSA, 3 major areas of investigation focused on biomarkers have emerged as essential for the field (which will undoubtedly require substantial discovery and validation efforts), namely: (i) diagnostic biomarkers: a panel of biological candidates that reliably discriminates between individuals with and without OSA; (ii) morbidity biomarkers: a panel of readily measurable biological products that reliably discerns among patients with OSA by detecting those at risk for developing or already present end-organ morbidities; (iii) treatment adherence/outcomes: biological approaches that identify those patients under higher risk of residual OSA or individuals with a differential response to treatment that places them at risk for morbidity.

There are 2 general approaches that have been used to investigate the aforementioned issues in OSA: candidate-based approaches (biased) and "omics" screens (unbiased). Candidate-based genetic studies, for example, compare the frequency of gene variants thought to relate disease susceptibility in groups with and without OSA. On the other hand, high-throughput platforms enabling the investigation of specific domains of the cell/tissue machinery,-+ such as MS, microarrays, and next generation sequencing, are aimed at the universal detection of genes (genomics), messenger RNA (mRNA) (transcriptomics), proteins (proteomics), metabolites (metabolomics), and epigenetic modifications in a specific biological sample.

The gold standard tool to diagnose OSA-overnight polysomnography (PSG) [94] can lead to sleep, it is expensive and uncomfortable for patients with suspected OSA, and labor intensive for the polysomnographic technologist. Thus, development of more comfortable and easier tools as surrogates for PSG monitoring, or alternative tests is an urgent task, as the research developed so far has not led to the desirable results [31]. Metabolomics could be the key discipline for providing the suitable biomarker(s) for early detection of OSA, but scant research in this field has been developed so far. The study of both the most suitable sample and analytical technique should be the starting point in this search. The proposal of some authors about the analytical technique to be used shows the lack of analytical knowledge: MS or chromatography alone [31], as if they are alternatives (the former is a detection technique, and the latter a separation technique).

The early authors working in OSA-metabolomics were reluctant to use the name of the last of the great omics; therefore, they discussed the role of VOCs-most of them metabolites-but did not use the word metabolomics or metabonomics [95], despite the first definition of metabolomics dates from 2002 [96] and metabolites as such are discussed in some of the early articles; nevertheless, the name used in these studies was metabolic profiling.

Example of a search for metabolomics biomarkers of OSA is the pilot study developed by Ferrarini et al., who used plasma from patients with no severe and severe OSA, looked for characteristics metabolic profiles using LC-QTOF/MS, and found 14 statistically significant features belonging to a variety of categories such as lipids, amino acids, and peptides, among others [76]. A study was aimed at analyzing the correlation between sleepiness and urine metabolites of neurotransmitters involved in the arousal system. Six metabolites of dopamine, norepinephrine and serotonin were analyzed, with the conclusion that the first could identify sleepy patients with OSA. In particular, the overnight change of urine DOPAC $(3,4-$ dihydroxyphenylacetic acid) could indicate OSA patients with excessive daytime sleepiness [73]. The main limitation of this study was the absence of a normal control group, necessary to exclude possible confounding effects. A conclusion from the authors that is not understandable for analytical chemists is the concept of "technique": "the metabolomics used in the present study may be a technique that holds promise for future investigation of biomarkers of daytime sleepiness" [73]. The assumption of metabolomics as a technique has been widely and erroneously used by a number of authors.

Based on metabolomics, a number of studies has been devoted to search for and compare biomarkers in a variety of biological samples to differentiate patients with COPD syndrome from those with OSA syndrome (taking into account that the latter occurs together with the former in $10-20 \%$ of the patients) $[54,97,98]$.

A study, developed in 2015 and discussed in the previous section, investigated the possibility of diagnosing a patient with either COPD or the OSA syndrome using a set of selected metabolites and to determine whether the metabolites that are present in one type of biofluid (serum, $\mathrm{EBC}$ or urine) or whether a combination of metabolites that are present in two biofluids or whether a set of metabolites that are present in all three biofluids are necessary to correctly diagnose a patient [91]. 
Concerning specific metabolic pathways, tryptophan (TRP) metabolism has been studied as it results in nicotinamid and serotonin production, but also in the production of numerous intermediates such as kynurenine, anthranilic and kynurenic acids, 3-hydroxykynurenine, 3-hydroxyanthranilic and quinolinic acids, potentially linked to cardiovascular disease and cancer. Alterations of TRP are presumable related to OSA-related cardiovascular co-morbidities and might be one of the players in the relationship between cancer and intermittent hypoxia/OSA. More research in this direction is required to either confirm or reject change of TRP metabolites levels as potential biomarkers in OSA-related diseases [99]. General is the need to go ahead with the investigation on biologically meaningful biomarkers that are cost-effective, valid, and precise to accurately predict cardiovascular risk in OSA to inform personalized medicine strategies and potentially identify those most likely to benefit from therapy, thereby informing future clinical trials [100].

Recent studies involved serum samples on the co-existence of OSA and enhanced vascular inflammation in patients with coronary artery disease also lack of the presence of a control group, mandatory to ensure that whether or not a decrease in inflammatory markers occurs in all patients after a cardiovascular event, irrespective of CPAP treatment [74].

On the other hand, a large number of the research works devoted to comparison of the metabolites in the EBC of individuals with COPD involved healthy controls [101-104]. The research developed so far on breath samples (both $\mathrm{EB}$ and $\mathrm{EBC}$ ) looking for biomarkers of lung cancer [44-46] have provided metabolomics profiles that can be the basis for OSA studies with the same aim. Also of interest is the research in this direction based on sweat samples, and the comparison of the results obtained with those from $\mathrm{EBC}$ [105]. The no-invasiveness of the sampling, the proved presence of key differently expressed metabolites, and each time more accepted role of metabolomics in the search for biomarkers paved the way for the present growing development of breath- and sweat-based investigation.

\section{Required improvements and guidelines for OSA care}

Specialists and researchers on OSA coincide with the necessity for development of investigations addressed to a better knowledge of the disease, the ways for an early diagnosis, the genetic predisposition to suffer OSA, and so on. Lim and Pack proposed in 2016 [2] up to 8 issues to be dealt with in the future, namely:

1. Develop a personalized approach to the diagnosis and management of obstructive sleep apnea (OSA).

2. Establish biomarkers to assess efficacy of therapy for OSA.

3. Elucidate gene variants that determine risk for OSA.

4. Determine if identified clinical subtypes of OSA are generalizable and alter outcomes of care.

5. Broaden the application of telemedicine for patient assessment and follow-up.

6. Implement care management that includes participation of patients to improve outcomes of therapy.

7. Determine whether identification and treatment of OSA affect the rate of progression of neurodegeneration.

8. Evaluate whether identification and treatment of OSA alter outcomes of cancer.

Despite the desirability of achievement of these issues, it is clear that they are far from being attainable in an immediate future owing to,
Personalized approaches to the diagnosis and management of diseases are at present more a dream than an achievable reality.

- The existence of biomarkers to assess the efficacy of therapy for OSA requires the previous existence of unequivocal biomarkers for OSA detection (desirably preventive biomarkers or those at the early OSA development). Unequivocal OSA biomarkers make mandatory to know the influence on them of other diseases in close relationship with OSA that promote similar/opposite alterations of the biomarkers.

- Elucidation of gene variants involved in risk for OSA requires in depth epigenetics studies, and even metabolomics studies that provide valuable up-stream information [37].

- Identification of clinical subtypes of OSA would be closely related to the previous issue; thus involving epigenetics studies prior to determining whether or not the identified subtypes are generalizable.

- Broadening telemedicine is only applicable to very developed countries and, within them, to people with a given formation level. Alternatives for less fortunate countries should be designed.

- Patients participation in care management is always dependent on the formation level of them; therefore, only a fraction of the patients could be included in this issue.

- The effect of identification and/or treatment of OSA on progression of neurodegeneration should be addressed by integrative omics, as the metabolic evolution and kinetics of the drugs involved in the treatment could provide key information on their up-stream action.

- Evaluation of the alterations of other diseases by the actions for OSA identification and/or treatment is a matter that clearly should be addressed by metabolomics with assistance from other omics, if required.

In the authors' opinion, the excellent issues proposed by Lim and Pack should be preceded by the following others:

- The in depth knowledge of OSA biomarkers and the influence of other diseases on their values [106].

- The effect of treatment alternatives to CPAP in OSA and the effects of interventions on the cardiovascular and metabolic consequences of OSA should also be a matter of research.

- The validation of the biomarkers, which is of paramount importance as solid support for the future of OSA management.

- The design of dedicate portable equipment for OSA diagnostic and adherence to treatment should follow validation.

- The best characterization of the influence of sex (biological differences) and gender (social and cultural differences) on OSA development [107]. Despite these differences have been recognized in terms of disease susceptibility, severity, or response to treatment, their association with differences in gene expression, transduction pathways, body habitus, behavior, and pharmacological responses is a pending goal to be addressed by integrative omics on which biomedical researchers, social scientists and analytical chemists should collaborate.

- The more abundant research on EB and EBC as key samples in OSA and related diseases. Sweat can be a complementary sample in this field [65].

- The sampling standardization in dealing with these scantly used biofluids is also a pending issue. 
- The binomial provided information-costs of the research based on metabolomics and that based on up-stream omics should be revisited [92].

- The incipient studies on the role of gut microbiota in OSA and its treatment should be the matter of in-depth research [29].

- The role of metabolomics in P4 should also be established.

\section{Conclusion}

Many other investigations will result foreseeably from those here proposed and from those proposed by clinicians and researchers, who should be supported on methods designed by analytical chemists in the light of: (i) the sample (matrix-analyte interaction); (ii) the changes in the target analytes (particularly decreased in concentration that can difficult or hinder their detection and make mandatory preconcentration steps); (iii) the appropriate high-resolution equipment for analytes separation; (iv) the technique selected for detection and the concrete instrument based on it, which on-line connected with the separation unit should constitute the best analytical platform; (v) the most suitable treatment of the obtained data to provide the clinicians with the results in the proper form for clinical interpretation.

\section{Acknowledgments}

This research has been jointly financed by the Spanish Ministerio de Economía y Competitividad (CTQ2015-68813-R project) and the European Regional Development Fund/European Social Fund ("Investing in your future"). CIBER de Fragilidad y Envejecimiento Saludable is an initiative of ISCIII, Spain.

\section{Highlights}

- The present situation of the research on metabolomics-OSA is critically evaluated.

- Both positive and negative aspects are discussed and solutions to the latter proposed.

- The search for OSA biomarkers based on metabolomics is considered a promising line.

- Aspects that may help to improve metabolomics-OSA research are finally discussed.

\section{References}

1. Peppard PE, Young T, Barnet JH, Palta M, Hagen EW, et al. (2013) Original contribution increased prevalence of sleep-disordered breathing in adults, Am J Epidemiol 177: 1006-1014. [Crossref]

2. Lim DC, Pack AI (2017) Obstructive sleep apnea: update and future. Annu Rev Med 68: 99-112. [Crossref]

3. Blechner M, Willamson AA (2016) Consequences of obstructive sleep apnea in children. Curr Probl Pediatr Adolesc Health Care 46: 29-26. [Crossref]

4. Tan HL, Gozal D, Kheirandish-Gozal L (2013) Obstructive sleep apnea in children: a critical update. Nat Sci Sleep 5 109-123. [Crossref]

5. Kaditis AG, Alonso Alvarez ML, Boudewyns A, Alexopoulos EI, Ersu R, et al. (2016) Obstructive sleep disordered breathing in 2- to 18-year-old children: diagnosis and management. Eur Respir J 47: 69-94. [Crossref]

6. Bazzano LA, Hu T, Bertisch SM, Yao L, Harville EW, Gustat J, et al. (2016) Childhood obesity patterns and relation to middle-age sleep apnoea risk: the Bogalusa Heart Study. Pediatr Obes 11: 535-542. [Crossref]

7. Singh P, Somers VK (2016) Obstructive sleep apnea, diabetes, and obesity: partners in crime? Sleep Med 25: 162-163. [Crossref]

8. McNicholas WT (2008) Diagnosis of obstructive sleep apnea in adults. Proc Am Thorac Soc 5: 154-160. [Crossref]
9. Kushida CA, Littner MR, Morgenthaler T, Alessi CA, Bailey D, et al. (2005) Practice parameters for the indications for polysomnography and related procedures: an update for 2005. Sleep 28: 499-523. [Crossref]

10. Durán-Cantolla J, Aizpuru F, Martínez-Null C, Barbé-Illa F (2009) Obstructive sleep apnea/hypopnea and systemic hypertension. Sleep Med Rev 13: 323-331. [Crossref]

11. Marin JM, Carrizo SJ, Vicente E, Agusti AGN (2005) Long-term cardiovascular outcomes in men with obstructive sleep apnoea-hypopnoea with or without treatment with continuous positive airway pressure: an observational study. Lancet 365: 10461053. [Crossref]

12. Aronson D, Nakhleh M, Zeidan-Shwiri T, Mutlak M, Lavie P, et al. (2014) Clinica implications of sleep disordered breathing in acute myocardial infarction. PLoS One 9: e88878. [Crossref]

13. Yaggi HK, Concato J, Kernan WN, Lichtman JH, Brass LM, et al. (2005) Obstructive sleep apnea as a risk factor for stroke and death. $N$ Engl J Med 353: 2034-2041. [Crossref]

14. Li M, Hou WS, Zhang XW, Tang ZY (2014) Obstructive sleep apnea and risk of stroke: a meta-analysis of prospective studies. Int J Cardiol 172: 466-469. [Crossref]

15. Harsch IA, Schahin SP, Radespiel-Tröger M, Weintz O, Jahreiß H, et al. (2004) Continuous positive airway pressure treatment rapidly improves insulin sensitivity in patients with obstructive sleep apnea syndrome. Am J Respir Crit Care Med 169: 156-162. [Crossref]

16. Schahin SP, Nechanitzky T, Dittel C, Fuchs FS, Hahn EG, et al. (2008) Long-term improvement of insulin sensitivity during CPAP therapy in the obstructive sleep apnoea syndrome. Med Sci Monit 14: CR117-CR121. [Crossref]

17. Punjabi NM, Sorkin JD, Katzel LI, Goldberg AP, Schwartz AR, et al. (2002) Sleepdisordered breathing and insulin resistance in middle-aged and overweight men. Am J Respir Crit Care Med 165: 677-682. [Crossref]

18. Mary S M Ip, Lam B, Ng MMY, Lam WK, Tsang KWT, et al. (2002) Obstructive sleep apnea is independently associated with insulin resistance. Am J Respir Crit Care Med 165: 670-676. [Crossref]

19. Morgenstern M, Wang J, Beatty N, Batemarco T, Sica AL, et al. (2014) Obstructive sleep apnea: an unexpected cause of insulin resistance and diabetes. Endocrinol Metab Clin 43: 187-204. [Crossref]

20. Arısoy A, Sertoğullarından B, Ekin S, Özgökçe M, Bulut MD, Huyut MT, et al. (2016) Sleep apnea and fatty liver are coupled via energy metabolism. Med Sci Monit Int Med J Exp Clin Res 22: 908-913. [Crossref]

21. Punjabi NM (2008) The epidemiology of adult obstructive sleep apnea. Proc Am Thorac Soc 5: 136-143. [Crossref]

22. Yaffe K, Laffan AM, Harrison SL, Redline S, Spira AP, et al. (2011) Sleep-disordered breathing, hypoxia, and risk of mild cognitive impairment and dementia in older women. JAMA 306: 613-619. [Crossref]

23. Campos-Rodriguez F, Martinez-Garcia MA, Martinez M, Duran-Cantolla J, de la Peña $\mathrm{M}$, et al. (2013) Association between obstructive sleep apnea and cancer incidence in a large multicenter Spanish cohort. Am J Respir Crit Care Med 187: 99-105. [Crossref]

24. Weaver TE, Grunstein RR (2008) Adherence to continuous positive airway pressure therapy: the challenge to effective treatment. Proc Am Thorac Soc 5: 173-178. [Crossref]

25. Mcardle N, Devereux G, Heidarnejad H, Engleman HM, Mackay TW, et al. (1999) Long-term use of CPAP therapy for sleep apnea/hypopnea syndrome. Am J Respir Crit Care Med 159: 1108-1114. [Crossref]

26. Park JG, Morgenthaler TM, Gay PC (2013) Novel and emerging nonpositive airway pressure therapies for sleep apnea. Chest 144: 1946-1952. [Crossref]

27. Lim DC, Sutherland K, Cistulli PA, Pack AI (2017) P4 medicine approach to obstructive sleep apnoea. Respirology 22: 849-860. [Crossref]

28. Arnardottir ES, Mackiewicz M, Gislason T, Teff KL, Pack AI (2009) Molecula signatures of obstructive sleep apnea in adults: a review and perspective. Sleep 32: 447-470. [Crossref]

29. Xu H, Wang H, Guan J, Yi H, Qian Y, et al. (2017) Effects of continuous positive airway pressure on neurocognitive architecture and function in patients with obstructive sleep apnoea: study protocol for a multicentre randomised controlled trial. BMJ Open 7: 1-8. [Crossref]

30. Xu H, Zheng X, Qian Y, Guan J, Yi H, et al. (2016) Metabolomics profiling for obstructive sleep apnea and simple snorers. Sci Rep 6: 1-9. [Crossref] 
31. Xu H, Zheng X, Jia W, Yin S (2015) Chromatography/mass spectrometry-based biomarkers in the field of obstructive sleep apnea. Med (Baltimore) 94: e1541. [Crossref]

32. Kentsis A (2011) Challenges and opportunities for discovery of disease biomarkers using urine proteomics. Pediatr Int 53: 1-6. [Crossref]

33. Mena-Bravo A, Priego-Capote F, Luque de Castro MD (2016) Two-dimensional liquid chromatography coupled to tandem mass spectrometry for vitamin D metabolite profiling including the C3-epimer-25-monohydroxyvitamin D3. J Chromatogr A 1451: 50-57. [Crossref]

34. López-Bascón MA, Priego-Capote F, Peralbo-Molina A, Calderón-Santiago M, Luque de Castro MD (2016) Influence of the collection tube on metabolomic changes in serum and plasma. Talanta 150: 681-689. [Crossref]

35. Mena-Bravo A, Calderón-Santiago M, Luque de Castro MD, Priego-Capote F (2018) Stability study for analysis of vitamin D and metabolites in human serum by liquid chromatography coupled to tandem mass spectrometry.

36. Decramer S, de Peredo AG, Breuil B, Mischak H, Monsarrat B, et al. (2008) Urine in clinical proteomics. Mol Cell Proteomics 7: 1850-1862. [Crossref]

37. Fernández-Peralbo MA, Luque de Castro MD (2012) Preparation of urine samples prior to targeted or untargeted metabolomics mass-spectrometry analysis. Trends Anal Chem 41: 75-85. [Crossref]

38. León-González Z, Ferreiro-Vera C, Priego-Capote F, Luque de Castro MD (2011) Targeting metabolomics analysis of the sunscreen agent 2-ethylhexyl 4-(N, Ndimethylamino) benzoate in human urine by automated on-line solid-phase extractionliquid chromatography-tandem mass spectrometry with liquid chromatography-timeof-flight/mass sp. J Chromatogr A 1218: 3013-3021. [Crossref]

39. Vaidyanathan K (2015) Urinary proteomics and metabolomics in the diagnosis of pediatric disorders. Proteomics Clin Appl 9: 482-489. [Crossref]

40. Hortin GL, Sviridov D (2007) Diagnostic potential for urinary proteomics. Pharmacogenomics 8: 237-255. [Crossref]

41. Tripathi A, Melnik AV, Xue J, Poulsen O, Meehan M, et al. (2018) Intermittent hypoxia and hypercapnia, a hallmark of obstructive sleep apnea, alters the gut microbiome and metabolome. mSystems 3: 1-9. [Crossref]

42. Michael DJ, Valle B, Cox J, Kalns JE, Fogt DL (2013) Salivary biomarkers of physical fatigue as markers of sleep deprivation. J Clin Sleep Med 9: 1325-1331. [Crossref]

43. Teranishi R, Mon TR, Robinson AB, Cary P, Pauling L (1972) Gas chromatography of volatiles from breath and urine. Anal Chem 44: 18-20. [Crossref]

44. Luque de Castro MD, Fernandez-Peralbo MA (2012) Analytical methods based on exhaled breath for early detection of lung cancer. Trends Anal Chem 38: 13-20. [Crossref]

45. Peralbo-Molina A, Calderón-Santiago M, Priego-Capote F, Jurado-Gámez B, Luque de Castro MD (2016) Metabolomics analysis of exhaled breath condensate for discrimination between lung cancer patients and risk factor individuals. J Breath Res 10: 16011. [Crossref]

46. Peralbo-Molina A, Calderón-Santiago M, Priego-Capote F, Jurado-Gámez B, Luque de Castro MD (2016) Identification of metabolomics panels for potential lung cancer screening by analysis of exhaled breath condensate. J Breath Res 10: 26002. [Crossref]

47. Schwarz EI, Engler A, Kohler M (2017) Exhaled breath analysis in obstructive sleep apnea. Expert Rev Respir Med 11: 631-639. [Crossref]

48. Horvath I, Hunt J, Barnes PJ, Alving K, Antczak A, et al. (2005) Exhaled breath condensate: methodological recommendations and unresolved questions. Eur Respir J 26: 523-548. [Crossref]

49. Rosias P (2012) Methodological aspects of exhaled breath condensate collection and analysis. J Breath Res 6: 27102. [Crossref]

50. Horváth I, Barnes PJ, Loukides S, Sterk PJ, Högman M, et al. (2017) A European respiratory society technical standard: exhaled biomarkers in lung disease. Eur Respir J 49: 1600965. [Crossref]

51. Pereira J, Porto-Figueira P, Cavaco C, Taunk K, Rapole S, et al. (2015) Breath analysis as a potential and non-invasive frontier in disease diagnosis: an overview. Metabolites 5: 3-55. [Crossref]

52. Beale D, Jones O, Karpe A, Dayalan S, Oh D, et al. (2016) A review of analytical techniques and their application in disease diagnosis in breathomics and salivaomics research. Int J Mol Sci 18: 24. [Crossref]
53. Amann A, Miekisch W, Pleil J, Risby T, Schubert J (2010) Methodological issues of sample collection and analysis of exhaled breath in volatile organic compounds. Maney Publishing, Leeds, UK, 49: 96-114. [Crossref]

54. Scarlata S, Pennazza G, Santonico M, Santangelo S, Rossi Bartoli I, et al. (2017) Screening of obstructive sleep apnea syndrome by electronic-nose analysis of volatile organic compounds. Sci Rep 7: 1-8. [Crossref]

55. Sibila O, Garcia-Bellmunt L, Giner J, Merino JL, Suarez-Cuartin G, et al. (2014) Identification of airway bacterial colonization by an electronic nose in chronic obstructive pulmonary disease. Respir Med 108: 1608-1614. [Crossref]

56. Lewis JM, Savage RS, Beeching NJ, Beadsworth MBJ, Feasey N, et al. (2017) Identifying volatile metabolite signatures for the diagnosis of bacterial respiratory tract infection using electronic nose technology: a pilot study. PLoS One 12: e0188879. [Crossref]

57. Rattray NJW, Hamrang Z, Trivedi DK, Goodacre R, Fowler SJ (2014) Taking your breath away: metabolomics breathes life in to personalized medicine. Trends Biotechnol 32: 538-548. [Crossref]

58. Smith D, Španěl P, Herbig J, Beauchamp J (2014) Mass spectrometry for real-time quantitative breath analysis. J Breath Res 8: 27101. [Crossref]

59. Fens N, Van der Schee MP, Brinkman P, Sterk PJ (2013) Exhaled breath analysis by electronic nose in airways disease. Established issues and key questions. Clin Exp Allergy 43: 705-715. [Crossref]

60. Hoffmeyer F, Raulf-Heimsoth M, Harth V, Bünger J, Brüning T (2009) Comparative analysis of selected exhaled breath biomarkers obtained with two different temperaturecontrolled devices. BMC Pulm Med 9: 48. [Crossref]

61. van der Schee M, Pinheiro H, Gaude E (2018) Breath biopsy for early detection and precision medicine in cancer. Ecancermedicalscience 12: ed84. [Crossref]

62. Peralbo-Molina A, Calderón-Santiago M, Priego-Capote F, Jurado-Gámez B Luque de Castro MD (2015) Development of a method for metabolomic analysis of human exhaled breath condensate by gas chromatography-mass spectrometry in high resolution mode. Anal Chim Acta 887: 118-126. [Crossref]

63. Fernandez-Peralbo MA, Santiago MC, Priego-Capote F, Luque de Castro MD (2015) Study of exhaled breath condensate sample preparation for metabolomics analysis by LC-MS/MS in high resolution mode. Talanta 144: 1360-1369. [Crossref]

64. Peralbo-Molina A, Calderón-Santiago M, Jurado-Gámez B, Luque de Castro MD Priego-Capote F (2017) Exhaled breath condensate to discriminate individuals with different smoking habits by GC-TOF/MS. Sci Rep 7: 1421. [Crossref]

65. Mena-Bravo A, Luque de Castro MD (2014) Sweat: a sample with limited presen applications and promising future in metabolomics. J Pharm Biomed Anal 90: 139147. [Crossref]

66. Luque de Castro MD (2016) Sweat as a clinical sample: what is done and what should be done? Bioanalysis 8: 85-88. [Crossref]

67. Delgado-Povedano MM, Calderón-Santiago M, Priego-Capote F, Luque de Castro MD (2016) Study of sample preparation for quantitative analysis of amino acids in human sweat by liquid chromatography-tandem mass spectrometry. Talanta 146: 310-317. [Crossref]

68. Delgado-Povedano MM, Calderón-Santiago M, Luque de Castro MD, Priego-Capote F (2018) Metabolomics analysis of human sweat collected after moderate exercise. Talanta 177: 47-65. [Crossref]

69. Delgado-Povedano MM, Castillo-Peinado LS, Calderón-Santiago M, Luque de Castro MD, Priego-Capote F (2020) Dry sweat as sample for metabolomics analysis. Talanta 288: 120428. [Crossref]

70. Luque de Castro MD, Luque García JL (2002) Acceleration and Automatization of Solid Sample Pretreatment. First Edit, Elsevier, Amsterdam, Netherlands. [Crossref]

71. Luque de Castro MD, Priego-Capote F (2007) Analytical Applications of Ultrasound, Elsevier, Oxford. [Crossref]

72. Fortis S, Lusczek ER, Weinert CR, Beilman GJ (2017) Metabolomics in COPD acute respiratory failure requiring noninvasive positive pressure ventilation. Can Respir $J$ 2017: 1-9. [Crossref]

73. Paik MJ, Kim DK, Nguyen DT, Lee G, Rhee CS, et al. (2014) Correlation of daytime sleepiness with urine metabolites in patients with obstructive sleep apnea. Sleep Breath 18: 517-523. [Crossref]

74. Thunström E, Glantz H, Yucel-Lindberg T, Lindberg K, Saygin M, et al. (2017) CPAP does not reduce inflammatory biomarkers in patients with coronary artery disease and nonsleepy obstructive sleep apnea: a randomized controlled trial. Sleep 40: 1-9. [Crossref] 
75. Cho K, Yoon DW, Lee M, So D, Hong IH, et al. (2017) Urinary metabolomic signatures in obstructive sleep apnea through targeted metabolomic analysis: a pilot study. Metabolomics 13: 1-9. [Crossref]

76. Ferrarini A, Rupérez FJ, Erazo M, Martínez MP, Villar-Álvarez F, et al. (2013) Fingerprinting-based metabolomic approach with LC-MS to sleep apnea and hypopnea syndrome: a pilot study. Electrophoresis 34: 2873-2881. [Crossref]

77. Barceló A, Bauça JM, Peña-Zarza JA, Morell-Garcia D, Yáñez A, et al. (2017) Circulating branched-chain amino acids in children with obstructive sleep apnea. Pediatr Pulmonol 52: 1085-1091. [Crossref]

78. Müller MJ, Volmer DA (2015) Mass spectrometric profiling of vitamin D metabolites beyond 25-hydroxyvitamin D. Clin Chem 61: 1033-1048. [Crossref]

79. Westhoff M, Litterst P (2012) Obstruktive schlafapnoe und oxidativer stress. Pneumologie 66: 610-615.

80. Incalzi RA, Pennazza G, Scarlata S, Santonico M, Vernile C, et al. (2015) Comorbidity modulates non invasive ventilation-induced changes in breath print of obstructive sleep apnea syndrome patients. Sleep Breath 19: 623-630. [Crossref]

81. Dragonieri S, Quaranta VN, Carratu P, Ranieri T, Resta O (2016) Exhaled breath profiling in patients with COPD and OSA overlap syndrome: a pilot study. $J$ Breath Res 10: 41001. [Crossref]

82. Li Y, Chongsuvivatwong V, Geater A, Liu A (2008) Are biomarker levels a good follow-up tool for evaluating obstructive sleep apnea syndrome treatments? Respiration 76: 317-323. [Crossref]

83. Dragonieri S, Porcelli F, Longobardi F, Carratù P, Aliani M, et al. (2015) An electronic nose in the discrimination of obese patients with and without obstructive sleep apnoea. J Breath Res 9: 26005. [Crossref]

84. Kunos L, Bikov A, Lazar Z, Korosi BZ, Benedek P, et al. (2015) Evening and morning exhaled volatile compound patterns are different in obstructive sleep apnoea assessed with electronic nose. Sleep Breath 19: 247-253. [Crossref]

85. Olopade CO, Christon JA, Zakkar M, Swedler WI, Rubinstein I, et al. (1997) Exhaled pentane and nitric oxide levels in patients with obstructive sleep apnea. Chest 111: 1500-1504. [Crossref]

86. Bayrakli I, Öztürk O, Akman H (2016) Investigation of acetone, butanol and carbon dioxide as new breath biomarkers for convenient and noninvasive diagnosis of obstructive sleep apnea syndrome. Biomed Chromatogr 30: 1890-1899. [Crossref]

87. Aoki T, Nagaoka T, Kobayashi N, Kurahashi M, Tsuji C, et al. (2017) Prospective analyses of volatile organic compounds in obstructive sleep apnea patients. Toxicol $\mathrm{Sci}$ 156: 362-374. [Crossref]

88. Schwarz EI, Sinues PML, Bregy L, Gaisl T, Gomez DG, et al. (2016) Effects of CPAP therapy withdrawal on exhaled breath pattern in obstructive sleep apnoea. Thorax 71 : 110-117. [Crossref]

89. Ruszkiewicz DM, Sanders D, O’Brien R, Hempel F, Reed MJ, et al. (2020) Diagnosis of COVID-19 by analysis of breath with gas chromatography-ion mobility spectrometry - a feasibility study. EClinicalMedicine 000: 100609. [Crossref]

90. Wang L, Tang Y, Liu S, Mao S, Ling Y, et al. (2013) Metabonomic profiling of serum and urine by $1 \mathrm{H}$ NMR-based spectroscopy discriminates patients with chronic obstructive pulmonary disease and healthy individuals. PLoS One 8: e65675. [Crossref]
91. Ząbek A, Stanimirova I, Deja S, Barg W, Kowal A, et al. (2015) Fusion of the 1 H NMR data of serum, urine and exhaled breath condensate in order to discriminate chronic obstructive pulmonary disease and obstructive sleep apnea syndrome. Metabolomics 11: 1563-1574. [Crossref]

92. Rodriguez-Perez N, Schiavi E, Frei R, Ferstl R, Wawrzyniak P, et al. (2017) Altered fatty acid metabolism and reduced stearoyl-coenzyme a desaturase activity in asthma. Eur J Allergy Clin Immunol 72: 1744-1752. [Crossref]

93. Gozal D, Kheirandish-Gozal L (2010) New approaches to the diagnosis of sleepdisordered breathing in children. Sleep Med 11: 708-713. [Crossref]

94. Berry RB, Budhiraja R, Gottlieb DJ, Gozal D, Iber C, et al. (2012) Rules for scoring respiratory events in sleep: update of the 2007 AASM manual for the scoring of sleep and associated events. J Clin Sleep Med 8: 597-619. [Crossref]

95. D. Gozal, Serum, urine, and breath-related biomarkers in the diagnosis of obstructive sleep apnea in children: is it for real?, Curr. Opin. Pulm. Med. 18 (2012) 561-567. doi:10.1097/MCP.0b013e328358be2d. [Crossref]

96. Fiehn O (2002) Metabolomics - the link between genotypes and phenotypes. Plant Mol Biol 48: 155-171. [Crossref]

97. McClay JL, Adkins DE, Isern NG, O'Connell TM, Wooten JB, et al. (2010) ${ }^{1} \mathrm{H}$ nuclear magnetic resonance metabolomics analysis identifies novel urinary biomarkers for lung function. J Proteome Res 9: 3083-3090. [Crossref]

98. Ubhi BK, Riley JH, Shaw PA, Lomas DA, Tal-Singer R, et al. (2012) Metabolic profiling detects biomarkers of protein degradation in COPD patients. Eur Respir $J 40$ 345-355. [Crossref]

99. Boulet L, Flore P, Le Gouellec A, Toussaint B, Pépin JL, et al. (2015) Is tryptophan metabolism involved in sleep apnea-related cardiovascular co-morbidities and cancer progression? Med Hypotheses 85: 415-423. [Crossref]

100. Mehra R (2017) Building evidence implicating novel cardiovascular biomarkers in obstructive sleep apnea. J Clin Sleep Med 13: 361-362. [Crossref]

101. Bertini I, Luchinat C, Miniati M, Monti S, Tenori L (2014) Phenotyping COPD by $1 \mathrm{H}$ NMR metabolomics of exhaled breath condensate. Metabolomics 10:302-311. [Crossref]

102. Basanta M, Ibrahim B, Dockry R, Douce D, Morris M, et al. (2012) Exhaled volatile organic compounds for phenotyping chronic obstructive pulmonary disease: a crosssectional study. Respir Res 13: 72. [Crossref]

103. Fens N, de Nijs SB, Peters S, Dekker T, Knobel HH, et al. (2011) Exhaled air molecular profiling in relation to inflammatory subtype and activity in COPD. Eur Respir J 38: 1301-1309. [Crossref]

104. de Laurentiis G, Paris D, Melck D, Maniscalco M, Marsico S, et al. (2008) Metabonomic analysis of exhaled breath condensate in adults by NMR spectroscopy. Eur Respir J 32: 1175-1183. [Crossref]

105. Calderón-Santiago M, Priego-Capote F, Turck N, Robin X, Jurado-Gámez B, et al. (2015) Human sweat metabolomics for lung cancer screening. Anal Bioanal Chem 407: 5381-5392. [Crossref]

106. Gulcev M, Reilly C, Griffin TJ, Broeckling CD, Sandri BJ, et al. (2016) Tryptophan catabolism in acute exacerbations of chronic obstructive pulmonary disease. Int $J$ Chron Obstruct Pulmon Dis 11: 2435 -2446. [Crossref]

107. List C, Han MK, Arteaga-solis E, Blenis J, Bourjeily G, et al. (2018) Female sex and gender in lung/sleep health and disease: increased understanding of basic biological, pathophysiological and behavioral mechanisms leading to better health for female patients with lung disease. AJRCCM 198: 1-28.

Copyright: (C2020 Castillo-Peinado LS. This is an open-access article distributed under the terms of the Creative Commons Attribution License, which permits unrestricted use, distribution, and reproduction in any medium, provided the original author and source are credited. 